\title{
Synthesis, in vitro anticancer screening and radiosensitizing evaluation of some new 4-[3-(substituted)thioureido]- $N$-(quinoxalin-2-yl)- benzenesulfonamide derivatives
}

\author{
MOSTAFA M. GHORAB ${ }^{1,3}$ \\ FATMA A. RAGAB ${ }^{2}$ \\ HELMY I. HEIBA ${ }^{3}$ \\ MARWA G. EL-GAZZAR ${ }^{3 *}$ \\ MOSTAFA G. EL-GAZZAR ${ }^{3}$ \\ ${ }^{1}$ Medicinal, Aromatic and Poisonous \\ Plants Research Center (MAPPRC) \\ College of Pharmacy, King Saud \\ University, Riyadh, Saudi Arabia \\ ${ }^{2}$ Department of Pharmaceutical \\ Chemistry, Faculty of Pharmacy \\ Cairo University, Cairo, Egypt \\ ${ }^{3}$ Department of Drug Radiation \\ Research, National Center for Radiation \\ Research and Technology, PO Box 29 \\ Nasr City, Cairo, Egypt
}

Accepted December 1, 2011

\begin{abstract}
Sulfonamides and quinoxaline derivatives possess many types of biological activities and have been recently reported to show substantial antitumor activity. This paper reports the synthesis of novel thioureido sulfaquinoxaline derivatives. All the newly synthesized compounds were evaluated for their in vitro anticancer activity against a human liver cell line (HEPG2) and showed higher activity than the reference drug doxorubicin. 4-(3-(4-Ethylbenzoate)thioureido)- $N$-(quinoxalin-2-yl)benzenesulfonamide (9) $\left(I C_{50}=15.6 \mu \mathrm{mol} \mathrm{L}-1\right), N$-(pyridin-2-yl)-4-(3-(4- $(N-$ -quinoxalin-2-yl-sulfamoyl)phenyl)thioureido)benzenesulfonamide (10) $\left(I C_{50}=26.8 \mu \mathrm{mol} \mathrm{L}^{-1}\right)$ and $N$-(quinoxalin-2-yl)-4-(3-(4-(N-thiazol-2-ylsulfamoyl)phenyl)thioureido)benzenesulfonamide (11) $\left(I C_{50}=24.4 \mu \mathrm{mol} \mathrm{L}^{-1}\right)$ were the most potent compared to doxorubicin $\left(I C_{50}=71.8\right.$ $\left.\mu \mathrm{mol} \mathrm{L}{ }^{-1}\right)$. The most potent compounds 9, 10 and 11 were evaluated as radiosensitizing agents by subjecting the compounds to $\gamma$-irradiation ( $8 \mathrm{kGy}$ ).
\end{abstract}

Keywords: quinoxaline, sulfonamides, anticancer activity, radiosensitizing effect

Quinoxaline derivatives show very interesting biological properties such as antibacterial (1), antiviral (2), antifungal (3), anthelmintic (4) and insecticidal (5). They were proven to be efficient anticancer agents when tested against several cell lines. Several quinoxaline derivatives were reported to be potent and highly selective epidermal growth factor receptor tyrosine kinase inhibitors (7). On the other hand, thioureido sulfonamide derivatives were reported to show significant anticancer activity by acting as carbonic anhydrase inhibitors (8). In the light of these facts and as a continuation of our previous work (9-12), we planned to synthesize novel thioureido sulfaquinoxaline

\footnotetext{
* Correspondence; e-mail: marwagalalgazzar@yahoo.com
} 
derivatives hoping that the new compounds might show significant anticancer activity and to study their structure-activity relationships. Moreover, this research was also aimed to evaluate the new compounds for their in vitro anticancer activity in combination with $\gamma$-irradiation.

\section{EXPERIMENTAL}

Melting points are uncorrected and were determined on a Stuart melting point apparatus (Stuart Scientific, UK). Elemental analyses $(\mathrm{C}, \mathrm{H}, \mathrm{N})$ were performed on a Perkin-Elmer 2400 analyzer (Perkin-Elmer, USA) at the Microanalytical Laboratories of the Faculty of Science, Cairo University. All compounds were within $\pm 0.4 \%$ of theoretical values. The IR spectra (KBr) were measured on a Shimadzu IR 110 Spectrophotometer (Shimadzu, Japan), ${ }^{1} \mathrm{H}$ NMR and ${ }^{13} \mathrm{C}$ NMR spectra were obtained with a Bruker proton NMR-Avance 300 instrument $(300 \mathrm{MHz})$ (Bruker, Germany), in DMSO- $d_{6}$ as a solvent, using tetramethylsilane (TMS) as internal standard. Mass spectra were run using an HP Model MS-5988 (Hewlett Packard, USA). All reactions were monitored with a thin layer chromatography using precoated aluminum sheets (Silica gel Merck $60 \mathrm{~F}_{254}$ ) and were visualized with a UV lamp (Merck, Germany). All chemicals (sulfaquinoxaline and doxorubicin) were commercially supplied from Sigma-Aldrich (USA).

\section{Syntheses}

4-Isothiocyanato-N-quinoxaline-2-yl-benzenesulfonamide (2). - To a suspension of sulfaquinoxaline $1(3 \mathrm{~g}, 0.01 \mathrm{~mol})$ in water $(30 \mathrm{~mL})$, thiophosgene $(1 \mathrm{~mL}, 0.01 \mathrm{~mol})$ was added and the reaction mixture was stirred for $1 \mathrm{~h}$ until red color of the thiophosgene disappeared and a white precipitate was formed. The precipitate was filtered off and washed with water to give compound 2 .

4-(3-Pyridin-2-yl-thioureido)-N-(quinoxalin-2-yl)benzenesulfonamide (3). - A mixture of $2(0.342 \mathrm{~g}, 0.01 \mathrm{~mol})$ and 2-aminopyridine $(0.094 \mathrm{~g}, 0.01 \mathrm{~mol})$ in dimethylformamide (DMF) (20 mL) containing 3 drops of triethylamine (TEA), was refluxed for $5 \mathrm{~h}$. The solid obtained was precipitated while hot and filtered to give compound 3 .

4-(3-(5-Chloropyridin-2-yl)thioureido)-N-(quinoxalin-2-yl)benzenesulfonamide (4). - A mixture of 2 (0.342 g, $0.01 \mathrm{~mol})$ and 2-amino-5-chloropyridine $(0.128 \mathrm{~g}, 0.001 \mathrm{~mol})$ in DMF (20 $\mathrm{mL}$ ), containing 3 drops of TEA, was refluxed for $5 \mathrm{~h}$. The solid obtained was precipitated while hot and filtered to give compound 4 .

4-(3-(5-Bromopyridin-2-yl)thioureido)-N-(quinoxalin-2-yl)benzenesulfonamide (5). - A mixture of $2(0.342 \mathrm{~g}, 0.001 \mathrm{~mol})$ and 2-amino-5-bromopyridine $(0.173 \mathrm{~g}, 0.001 \mathrm{~mol})$ in DMF $(20 \mathrm{~mL})$, containing 3 drops of TEA, was refluxed for $5 \mathrm{~h}$. The solid obtained was precipitated while hot and filtered to give compound 5.

4-(3-(4-Bromophenyl)thioureido)-N-(quinoxalin-2-yl)benzenesulfonamide (6). - The mixture of $2(0.342 \mathrm{~g}, 0.01 \mathrm{~mol})$ and $p$-bromoaniline $(0.171 \mathrm{~g}, 0.001 \mathrm{~mol})$ in dioxane $(20 \mathrm{~mL})$, with 3 drops of TEA, was refluxed for $1 \mathrm{~h}$, the solid obtained was precipitated when cold and filtered to give compound 6 . 
4-(3-(4-Fluorophenyl)thioureido)-N-(quinoxalin-2-yl)benzenesulfonamide (7). - A mixture of $2(0.342 \mathrm{~g}, 0.01 \mathrm{~mol})$ and $p$-fluoroaniline $(0.111 \mathrm{~g}, 0.001 \mathrm{~mol})$ in dioxane $(20 \mathrm{~mL})$, with 3 drops of TEA, was refluxed for $1 \mathrm{~h}$. The solid obtained was precipitated when cold and filtered to give compound 7.

4-(3-(4-Chlorophenyl)thioureido)-N-(quinoxalin-2-yl)benzenesulfonamide (8). - A mixture of $2(0.342 \mathrm{~g}, 0.001 \mathrm{~mol})$ and $p$-chloroaniline $(0.127 \mathrm{~g}, 0.001 \mathrm{~mol})$ in dioxane $(20 \mathrm{~mL})$, with 3 drops of TEA, was refluxed for $1 \mathrm{~h}$, the solid obtained was precipitated while hot and filtered to give compound 8 .

4-(3-(4-Carbethoxy)thioureido)-N-(quinoxalin-2-yl)benzenesulfonamide (9). - A mixture of $2(0.342 \mathrm{~g}, 0.001 \mathrm{~mol})$ and benzocaine $(0.165 \mathrm{~g}, 0.001 \mathrm{~mol})$ in dioxane $(20 \mathrm{~mL})$, with 3 drops of TEA, was refluxed for $5 \mathrm{~h}$. The solid obtained was precipitated when hot and filtered to give compound 9.

Table I. Physical and analytical data of newly synthesized compounds

\begin{tabular}{|c|c|c|c|c|c|c|}
\hline \multirow{2}{*}{ Compd. $^{a}$} & \multirow{2}{*}{$\begin{array}{l}\text { M.p. } \\
\left({ }^{\circ} \mathrm{C}\right)^{b}\end{array}$} & \multirow{2}{*}{$\begin{array}{l}\text { Yield } \\
(\%)\end{array}$} & \multirow{2}{*}{$\begin{array}{l}\text { Mol. formula } \\
\qquad\left(M_{\mathrm{r}}\right)\end{array}$} & \multicolumn{3}{|c|}{ Analysis calc. / found (\%) } \\
\hline & & & & $\mathrm{C}$ & $\mathrm{H}$ & $\mathrm{N}$ \\
\hline \multirow{2}{*}{2} & \multirow{2}{*}{$210-212$} & \multirow{2}{*}{86} & $\mathrm{C}_{15} \mathrm{H}_{10} \mathrm{~N}_{4} \mathrm{O}_{2} \mathrm{~S}_{2}$ & 52.62 & 2.94 & 16.36 \\
\hline & & & $(342.40)$ & 52.40 & 2.83 & 16.11 \\
\hline \multirow{2}{*}{3} & \multirow{2}{*}{$244-246$} & \multirow{2}{*}{83} & $\mathrm{C}_{20} \mathrm{H}_{16} \mathrm{~N}_{6} \mathrm{O}_{2} \mathrm{~S}_{2}$ & 55.03 & 3.69 & 19.25 \\
\hline & & & $(436.51)$ & 55.43 & 3.90 & 18.98 \\
\hline \multirow{2}{*}{4} & \multirow{2}{*}{$240-242$} & \multirow{2}{*}{91} & $\mathrm{C}_{20} \mathrm{H}_{15} \mathrm{ClN}_{6} \mathrm{O}_{2} \mathrm{~S}_{2}$ & 51.01 & 3.21 & 17.84 \\
\hline & & & $(470.96)$ & 50.90 & 2.85 & 17.50 \\
\hline \multirow{2}{*}{5} & \multirow{2}{*}{$246-248$} & \multirow{2}{*}{93} & $\mathrm{C}_{20} \mathrm{H}_{15} \mathrm{BrN}_{6} \mathrm{O}_{2} \mathrm{~S}_{2}$ & 46.61 & 2.93 & 16.31 \\
\hline & & & $(514.41)$ & 46.25 & 2.90 & 15.90 \\
\hline \multirow{2}{*}{6} & \multirow{2}{*}{$220-222$} & \multirow{2}{*}{85} & $\mathrm{C}_{21} \mathrm{H}_{16} \mathrm{BrN}_{5} \mathrm{O}_{2} \mathrm{~S}_{2}$ & 49.03 & 3.14 & 13.61 \\
\hline & & & $(513.42)$ & 49.35 & 3.50 & 13.85 \\
\hline \multirow{2}{*}{7} & \multirow{2}{*}{$177-180$} & \multirow{2}{*}{92} & $\mathrm{C}_{21} \mathrm{H}_{16} \mathrm{FN}_{5} \mathrm{O}_{2} \mathrm{~S}_{2}$ & 55.62 & 3.56 & 15.44 \\
\hline & & & $(453.51)$ & 55.15 & 3.48 & 15.39 \\
\hline \multirow{2}{*}{8} & \multirow{2}{*}{$128-130$} & \multirow{2}{*}{90} & $\mathrm{C}_{21} \mathrm{H}_{16} \mathrm{ClN}_{5} \mathrm{O}_{2} \mathrm{~S}_{2}$ & 53.67 & 3.43 & 14.90 \\
\hline & & & $(469.97)$ & 53.33 & 3.25 & 14.67 \\
\hline \multirow{2}{*}{9} & \multirow{2}{*}{$135-136$} & \multirow{2}{*}{89} & $\mathrm{C}_{24} \mathrm{H}_{21} \mathrm{~N}_{5} \mathrm{O}_{4} \mathrm{~S}_{2}$ & 56.79 & 4.17 & 13.80 \\
\hline & & & $(507.58)$ & 57.10 & 4.46 & 13.95 \\
\hline \multirow{2}{*}{10} & \multirow{2}{*}{$248-249$} & \multirow{2}{*}{87} & $\mathrm{C}_{26} \mathrm{H}_{21} \mathrm{~N}_{7} \mathrm{O}_{4} \mathrm{~S}_{3}$ & 52.78 & 3.58 & 16.57 \\
\hline & & & $(591.68)$ & 52.45 & 3.18 & 16.23 \\
\hline \multirow{2}{*}{11} & \multirow{2}{*}{$251-252$} & \multirow{2}{*}{94} & $\mathrm{C}_{24} \mathrm{H}_{19} \mathrm{~N}_{7} \mathrm{O}_{4} \mathrm{~S}_{4}$ & 48.23 & 3.20 & 16.40 \\
\hline & & & (597.71) & 48.44 & 3.38 & 16.60 \\
\hline
\end{tabular}

a Starting material $\mathbf{1}$ is not included in the table since it is commercially available.

b Crystallization solvent was ethanol. 
Table II. Spectral data of newly synthesized compounds

\begin{tabular}{|c|c|c|c|c|}
\hline Compd. $^{a}$ & $\begin{array}{c}\mathrm{IR} \\
\left(v, \mathrm{~cm}^{-1}\right)\end{array}$ & $\begin{array}{c}{ }^{1} \mathrm{H} \text { NMR }\left(\mathrm{DMSO}-d_{6}\right) \\
(\delta, \mathrm{ppm})\end{array}$ & $\begin{array}{c}{ }^{13} \mathrm{C} \text { NMR } \\
\left(\mathrm{DMSO}-d_{6}\right) \\
(\delta, \mathrm{ppm})\end{array}$ & MS \\
\hline 2 & $\begin{array}{l}3290(\mathrm{NH}) \\
3070(\mathrm{CH} \\
\text { arom. }), 2106 \\
(\mathrm{NCS}), 1330 \\
1156\left(\mathrm{SO}_{2}\right)\end{array}$ & $\begin{array}{l}4.0(\mathrm{~s}, 1 \mathrm{H}, \mathrm{NH} \text {, exchange- } \\
\left.\text { able with } \mathrm{D}_{2} \mathrm{O}\right), 7.01,7.5 \\
(2 \mathrm{~d}, 4 \mathrm{H}, \mathrm{Ar}-\mathrm{H} \mathrm{AB} \\
\text { system }), 7.68-8.05(\mathrm{~m}, \\
4 \mathrm{H}, \mathrm{Ar}-\mathrm{H}), 8.07(\mathrm{~s}, 1 \mathrm{H}, \\
\mathrm{CH} \text {-quinoxaline) }\end{array}$ & $\begin{array}{l}124.1,125.2, \\
126.9,128.9, \\
134.8,135.1 \\
135.6,136.8 \\
\text { (NCS), } 138.3 \\
161.9\end{array}$ & $\begin{array}{l}342\left(\mathrm{M}^{+}, 10.2 \%\right), \\
236(100 \%) \\
\left(\mathrm{C}_{10} \mathrm{H}_{11} \mathrm{~N}_{3} \mathrm{O}_{2} \mathrm{~S}\right. \\
N-(5,6 \text {-dihydro- } \\
\text {-pyrazin-2-yl)- } \\
\text {-benzenesulfon- } \\
\text { amide) }\end{array}$ \\
\hline 3 & $\begin{array}{l}3436,3360, \\
3252(3 \mathrm{NH}), \\
3072(\mathrm{CH} \\
\text { arom. } 1318, \\
1150\left(\mathrm{SO}_{2}\right) \\
1234(\mathrm{C}=\mathrm{S})\end{array}$ & $\begin{array}{l}4.0(\mathrm{~s}, 3 \mathrm{H}, \mathrm{NH} \text {, exchange- } \\
\left.\text { able with } \mathrm{D}_{2} \mathrm{O}\right), 6.5-6.7(\mathrm{~m}, \\
2 \mathrm{H}, 2 \mathrm{CH} \text { pyridine }), 6.74, \\
7.68(2 \mathrm{~d}, 4 \mathrm{H}, \mathrm{Ar}-\mathrm{H} \mathrm{AB} \text { sys- } \\
\text { tem }), 7.88-8.05(\mathrm{~m}, 4 \mathrm{H} \text {, } \\
\text { Ar-H), } 8.07(\mathrm{~s}, 1 \mathrm{H}, \mathrm{CH}- \\
\text {-quinoxaline }), 8.11,8.3(2 \mathrm{t} \text {, } \\
2 \mathrm{H}, 2 \mathrm{CH} \text { pyridine) }\end{array}$ & - & $\begin{array}{l}436\left(\mathrm{M}^{+}, 0.8 \%\right), \\
236(100 \%) \\
\left(\mathrm{C}_{10} \mathrm{H}_{11} \mathrm{~N}_{3} \mathrm{O}_{2} \mathrm{~S}\right. \\
N-(5,6 \text {-dihydro- } \\
\text {-pyrazin-2-yl)- } \\
\text {-benzenesulfon- } \\
\text { amide) }\end{array}$ \\
\hline 4 & $\begin{array}{l}3436,3358, \\
3246(3 \mathrm{NH}), \\
3070(\mathrm{CH} \\
\text { arom }), 1316, \\
1148\left(\mathrm{SO}_{2}\right) \\
1232(\mathrm{C}=\mathrm{S})\end{array}$ & $\begin{array}{l}4.0(\mathrm{~s}, 3 \mathrm{H}, \mathrm{NH} \text {, exchange- } \\
\left.\text { able with } \mathrm{D}_{2} \mathrm{O}\right), 6.74,7.68 \\
(2 \mathrm{~d}, 4 \mathrm{H}, \mathrm{Ar}-\mathrm{H} \mathrm{AB} \text { system }), \\
7.8-8.05(\mathrm{~m}, 4 \mathrm{H}, \text { Ar-H), } 8.1 \\
(\mathrm{s}, 1 \mathrm{H}, \mathrm{CH}-\text { quinoxaline), } \\
8.3 \text { (t, 3H, 3CH pyridine) }\end{array}$ & - & $\begin{array}{l}470\left(\mathrm{M}^{+}, 6.05 \%\right) \text {, } \\
84(100 \%) \\
\left(\mathrm{C}_{4} \mathrm{H}_{8} \mathrm{~N}_{2}, 1,2,3,6-\right. \\
\text {-tetrahydropyra- } \\
\text { zine) }\end{array}$ \\
\hline 5 & $\begin{array}{l}3338,3258, \\
3224(3 \mathrm{NH}), \\
3072(\mathrm{CH} \\
\text { arom. }), 1232 \\
(\mathrm{C}=\mathrm{S}), 1318, \\
1148\left(\mathrm{SO}_{2}\right)\end{array}$ & $\begin{array}{l}4.0(\mathrm{~s}, 3 \mathrm{H}, \mathrm{NH}, \text { exchan- } \\
\left.\text { geble with } \mathrm{D}_{2} \mathrm{O}\right), 6.4,7.6 \\
(2 \mathrm{~d}, 4 \mathrm{H}, \mathrm{Ar}-\mathrm{H} \mathrm{AB} \text { system), } \\
7.88-8.0(\mathrm{~m}, 4 \mathrm{H}, \text { Ar-H), } 8.1 \\
\text { (s, } 1 \mathrm{H}, \mathrm{CH}-q u i n o x a l i n e) \\
8.23(\mathrm{t}, 3 \mathrm{H}, 3 \mathrm{CH} \text { pyridine) }\end{array}$ & - & $\begin{array}{l}515\left(\mathrm{M}^{+}, 0.5 \%\right) \\
236(100 \%) \\
\left(\mathrm{C}_{10} \mathrm{H}_{11} \mathrm{~N}_{3} \mathrm{O}_{2} \mathrm{~S},\right. \\
N \text {-(5,6-dihydropy } \\
\text { razin-2-yl)-benze- } \\
\text { nesulfonamide) }\end{array}$ \\
\hline 6 & $\begin{array}{l}3442,3360, \\
3198(3 \mathrm{NH}), \\
3072(\mathrm{CH} \\
\text { arom }), 1236 \\
(\mathrm{C}=\mathrm{S}), 1314, \\
1148\left(\mathrm{SO}_{2}\right)\end{array}$ & $\begin{array}{l}4.0(\mathrm{~s}, 3 \mathrm{H}, \mathrm{NH}, \text { exchange- } \\
\left.\text { able with } \mathrm{D}_{2} \mathrm{O}\right), 6.35,7.18 \\
(2 \mathrm{~d}, 4 \mathrm{H}, \mathrm{Ar}-\mathrm{H} \mathrm{AB} \text { system }) \\
6.74,7.68(2 \mathrm{~d}, 4 \mathrm{H}, \mathrm{Ar}-\mathrm{H} \mathrm{AB} \\
\text { system }), 7.7-8.07(\mathrm{~m}, 4 \mathrm{H}, \\
\text { Ar-H), } 8.05 \text { (s, } 1 \mathrm{H}, \\
\mathrm{CH}-\text { quinoxaline) }\end{array}$ & - & $\begin{array}{l}513\left(\mathrm{M}^{+}, 0.8 \%\right), \\
149(100 \%) \\
\left(\mathrm{C}_{5} \mathrm{H}_{15} \mathrm{~N}_{3} \mathrm{~S}, \mathrm{~N}^{-}\right. \\
\text {-(methylthio) } \\
\text { piperazine-2- } \\
\text {-amine) }\end{array}$ \\
\hline 7 & $\begin{array}{l}3355,3314, \\
3212(3 \mathrm{NH}), \\
3016(\mathrm{CH} \\
\text { arom }), 1228 \\
(\mathrm{C}=\mathrm{S}), 1338 \\
1152\left(\mathrm{SO}_{2}\right)\end{array}$ & $\begin{array}{l}4.0(\mathrm{~s}, 3 \mathrm{H}, \mathrm{NH}, \text { exchange- } \\
\left.\text { able with } \mathrm{D}_{2} \mathrm{O}\right), 6.44,6.72 \\
(2 \mathrm{~d}, 4 \mathrm{H}, \mathrm{Ar}-\mathrm{H} \mathrm{AB} \text { system }) \\
6.74,7.68(2 \mathrm{~d}, 4 \mathrm{H}, \mathrm{Ar}-\mathrm{H} \mathrm{AB} \\
\text { system }), 7.72-8.1 \text { (m, } 4 \mathrm{H}, \\
\text { Ar-H), } 8.2(\mathrm{~s}, 1 \mathrm{H}, \mathrm{CH} \text {-quino- } \\
\text { xaline) }\end{array}$ & - & $\begin{array}{l}453\left(\mathrm{M}^{+}, 1.5 \%\right) \\
111(100 \%) \\
\left(\mathrm{C}_{5} \mathrm{H}_{9} \mathrm{~N}_{3}, 2,3-\right. \\
\text {-dihydro-2,6-di- } \\
\text { methylpyrazine) }\end{array}$ \\
\hline
\end{tabular}


M. M. Ghorab et al.: Synthesis, in vitro anticancer screening and radiosensitizing evaluation of some new 4-(3-(substituted)thioureido) -N-(quinoxalin-2-yl) benzenesulfonamide derivatives, Acta Pharm. 61 (2011) 415-426.

\begin{tabular}{|c|c|c|c|c|}
\hline 8 & $\begin{array}{l}3320,3236, \\
3163(3 \mathrm{NH}), \\
3072(\mathrm{CH} \\
\text { arom }), 1299 \\
(\mathrm{C}=\mathrm{S}), 1362, \\
1143\left(\mathrm{SO}_{2}\right)\end{array}$ & $\begin{array}{l}4.0(\mathrm{~s}, 3 \mathrm{H}, 3 \mathrm{NH} \text {, exchange- } \\
\left.\text { able with } \mathrm{D}_{2} \mathrm{O}\right), 6.4,7.02(2 \mathrm{~d}, \\
4 \mathrm{H}, \mathrm{Ar}-\mathrm{H} \mathrm{AB} \text { system }), 6.74, \\
7.68(2 \mathrm{~d}, 4 \mathrm{H}, \mathrm{Ar}-\mathrm{H} \mathrm{AB} \text { sys- } \\
\text { tem }), 7.78-8.1(\mathrm{~m}, 4 \mathrm{H}, \text { Ar- } \mathrm{H}) \text {, } \\
8.3 \text { (s, } 1 \mathrm{H}, \mathrm{CH} \text {-quinoxaline) }\end{array}$ & - & $\begin{array}{l}469\left(\mathrm{M}^{+}, 5 \%\right) \\
126(100 \%) \\
\left(\mathrm{C}_{7} \mathrm{H}_{14} \mathrm{~N}_{2}, N^{1-}\right. \\
\text {-methylcyclo- } \\
\text { hex-5-ene-1,2- } \\
\text { diamine) }\end{array}$ \\
\hline 9 & $\begin{array}{l}3510,3450, \\
3208(3 \mathrm{NH}), \\
3072(\mathrm{CH} \\
\text { arom }), 2992 \\
(\mathrm{CH} \text { aliph), } \\
1710(\mathrm{C}=0), \\
1282(\mathrm{C}=\mathrm{S}), \\
1310,1114 \\
\left(\mathrm{SO}_{2}\right)\end{array}$ & $\begin{array}{l}1.2\left(\mathrm{t}, 3 \mathrm{H}, \mathrm{CH}_{3}\right), 4.0(\mathrm{~s}, 3 \mathrm{H}, \\
3 \mathrm{NH}, \text { exchangeable with } \\
\left.\mathrm{D}_{2} \mathrm{O}\right), 4.3\left(\mathrm{q}, 2 \mathrm{H}, \mathrm{CH}_{2}\right), 6.57, \\
7.52(2 \mathrm{~d}, 4 \mathrm{H}, \mathrm{Ar}-\mathrm{H} \mathrm{AB} \text { sys- } \\
\text { tem }), 6.74,7.6(2 \mathrm{~d}, 4 \mathrm{vH}, \\
\mathrm{Ar}-\mathrm{H}, \mathrm{AB} \text { system }), 7.7-8.1 \\
(\mathrm{~m}, 4 \mathrm{H}, \text { Ar- } \mathrm{H}), 8.6(\mathrm{~s}, 1 \mathrm{H}, \\
\mathrm{CH} \text { quinoxaline })\end{array}$ & - & - \\
\hline 10 & $\begin{array}{l}3440,3360, \\
3250(3 \mathrm{NH}), \\
3070(\mathrm{CH} \\
\text { arom }), 1314, \\
1148\left(2 \mathrm{SO}_{2}\right)\end{array}$ & $\begin{array}{l}4.0(\mathrm{~s}, 4 \mathrm{H}, 4 \mathrm{NH} \text {, exchange- } \\
\left.\text { able with } \mathrm{D}_{2} \mathrm{O}\right), 6.4,7.02 \\
(2 \mathrm{~d}, 8 \mathrm{H}, \mathrm{Ar}-\mathrm{H} \mathrm{AB} \text { system), } \\
7.2-7.5(\mathrm{~m}, 4 \mathrm{H}, 4 \mathrm{CH}-\text { pyri- } \\
\text { dine }), 7.8-8.1(\mathrm{~m}, 4 \mathrm{H}, \text { Ar- } \mathrm{H}) \text {, } \\
8.2 \text { (s, } 1 \mathrm{H}, \mathrm{CH} \text {-quinoxaline) }\end{array}$ & - & $\begin{array}{l}591\left(\mathrm{M}^{+}, 0.8 \%\right), \\
264(29.93 \%), \\
111(100 \%) \\
\left(\mathrm{C}_{5} \mathrm{H}_{9} \mathrm{~N}_{3},\right. \\
\text { 2,3-dihydro-2,6- } \\
\text { dimethyl- } \\
\text { pyrazine) }\end{array}$ \\
\hline 11 & $\begin{array}{l}3436,3360, \\
3252(3 \mathrm{NH}), \\
3072(\mathrm{CH} \\
\text { arom }), 1264 \\
(\mathrm{C}=\mathrm{S}), 1310, \\
1148\left(\mathrm{SO}_{2}\right)\end{array}$ & $\begin{array}{l}4.0(\mathrm{~s}, 4 \mathrm{H}, 4 \mathrm{NH} \text {, exchange- } \\
\left.\text { able with } \mathrm{D}_{2} \mathrm{O}\right), 6.74,7.68 \\
(2 \mathrm{~d}, 8 \mathrm{H}, \mathrm{Ar}-\mathrm{H} \mathrm{AB} \text { system }) \\
7.7-8.02(\mathrm{~m}, 4 \mathrm{H}, \text { Ar-H), } 8.2, \\
8.32(2 \mathrm{~d}, 2 \mathrm{H}, 2 \mathrm{CH}-\text { thiazole }) \\
8.4(\mathrm{~s}, 1 \mathrm{H}, \mathrm{CH}-\text { quinoxaline })\end{array}$ & - & $\begin{array}{l}597\left(\mathrm{M}^{+}, 3 \%\right), \\
236(100 \%) \\
\left(\mathrm{C}_{10} \mathrm{H}_{11} \mathrm{~N}_{3} \mathrm{O}_{2} \mathrm{~S},\right. \\
N-(5,6 \text {-dihydro- } \\
\text { pyrazin-2-yl)- } \\
\text {-benzenesulfon- } \\
\text { amide) }\end{array}$ \\
\hline
\end{tabular}

a Starting material $\mathbf{1}$ is not included in the table since it is commercially available.

N-(Pyridin-2-yl)-4-(3-(4-(N-quinoxalin-2-yl-sulfamoyl)phenyl)thioureido)benzenesulfonamide (10). - A mixture of $2(0.342 \mathrm{~g}, 0.01 \mathrm{~mol})$ and sulfapyridine $(0.233 \mathrm{~g}, 0.01 \mathrm{~mol})$ in DMF $(20 \mathrm{~mL})$, with 3 drops of TEA, was refluxed for $5 \mathrm{~h}$. The solid was precipitated when hot and filtered to give compound $\mathbf{1 0}$.

N-(Quinoxalin-2-yl)-4-(3-(4-(N-thiazol-2-ylsulfamoyl)phenyl)thioureido)benzenesulfonamide (11). - A mixture of $2(0.342 \mathrm{~g}, 0.001 \mathrm{~mol})$ and sulfathiazole $(0.239 \mathrm{~g}, 0.001 \mathrm{~mol})$ in DMF $(20 \mathrm{~mL})$, with 3 drops of TEA, was refluxed for $5 \mathrm{~h}$. The solid was precipitated when hot and filtered to give compound $\mathbf{1 1}$.

\section{In vitro anticancer screening}

The human tumor cell line (HEPG2) was obtainedfrom the National Cancer Institute, Cairo, Egypt. Irradiation was performed using a Gamma cell-40 $\left({ }^{60} \mathrm{Co}\right)$ source 
[radioactivity of one gram of ${ }^{60} \mathrm{Co}$ is $44 \mathrm{TBq}$ (about 1100 Curies)]. The antitumor activity of the newly synthesized compounds was measured by the sulfo-rhodamine-B stain (SRB) assay as reported by Skehan et al. (13). Cells were plated in 96-multiwell plates ( $10^{4}$ cells per well) for $24 \mathrm{~h}$ before treatment with the compounds to allow attachment of cells to the plate wall. Tested compounds were dissolved and diluted with DMSO. Different concentrations of the compounds under test $\left(5,12.5,25\right.$ and $\left.40 \mu \mathrm{mol} \mathrm{L}^{-1}\right)$ were added to the cell monolayer. Triplicate wells were prepared for each individual dose. Monolayer cells were incubated with the compounds for $48 \mathrm{~h}$ at $37{ }^{\circ} \mathrm{C}$ and in an atmosphere of $5 \% \mathrm{CO}_{2}$. After $48 \mathrm{~h}$, cells were fixed, washed and stained for 30 min with 0.4 $\%(\mathrm{~m} / \mathrm{V})$ SRB dissolved in $1 \%$ acetic acid. Unbounded dye was removed through four washes with $1 \%$ acetic acid, and attached stain was recovered with Tris-EDTA buffer. Color intensity was measured in an ELISA reader (SPR-960B Sunostick Medical Technology, UK). Negative control was added using cell lines with the solvent without the drug. The relation between the surviving fraction and drug concentration was plotted to get the survival curve of each tumor cell line after a specified time. The concentration required for $50 \%$ inhibition of cell viability $\left(I C_{50}\right)$ was calculated and compared with the reference drug doxorubicin and the results are given in Table III.

\section{Radiosensitizing activity}

The most potent compounds resulting from the in vitro anticancer screening (compounds 9, 10 and 11) were selected in order to evaluate their in vitro anticancer activity in combination with $\gamma$-radiation. This study was conducted to evaluate the ability of these compounds to enhance the cell killing effect of $\gamma$-radiation. Negative control was added using cell lines with the solvent without the drug and radiation alone. Cells were subjected to a single dose of $\gamma$-irradiation at a dose level of $8 \mathrm{kGy}$ with a dose rate of 2 $\mathrm{kGy} \mathrm{min}^{-1}$. The surviving fractions were expressed as the mean \pm standard error. The results were analyzed using the 1-way ANOVA test and are given in Table IV.

\section{RESULTS AND DISCUSSION}

\section{Chemistry}

4-Isothiocyanato- $N$-quinoxaline-2-yl-benzene sulfonamide 2 was prepared by the reaction of $\mathrm{N}$-quinoxaline-2-yl-benzenesulfonamide with thiophosgene as reported earlier (14). Compound 2 was used to prepare different sulfonamide derivatives by incorporating different biologically active moieties. Compound 2 reacted with 2 -aminopyridine to obtain the corresponding thioureido derivative 3 , while its interaction with 2-aminopyridine, 2-amino-5-chloropyridine and 2-amino-5-bromopyridine yielded compounds 4 and 5, respectively (Scheme 1).

The isothiocyanato derivative 2 was further reacted with different aniline derivatives to obtain the corresponding thioureido derivatives. Thus, its interaction with 4-bromoaniline, 4-fluoroaniline, 4-chloroaniline and benzocaine yielded compounds 6, 7, 8, and $\mathbf{9}$, respectively. 


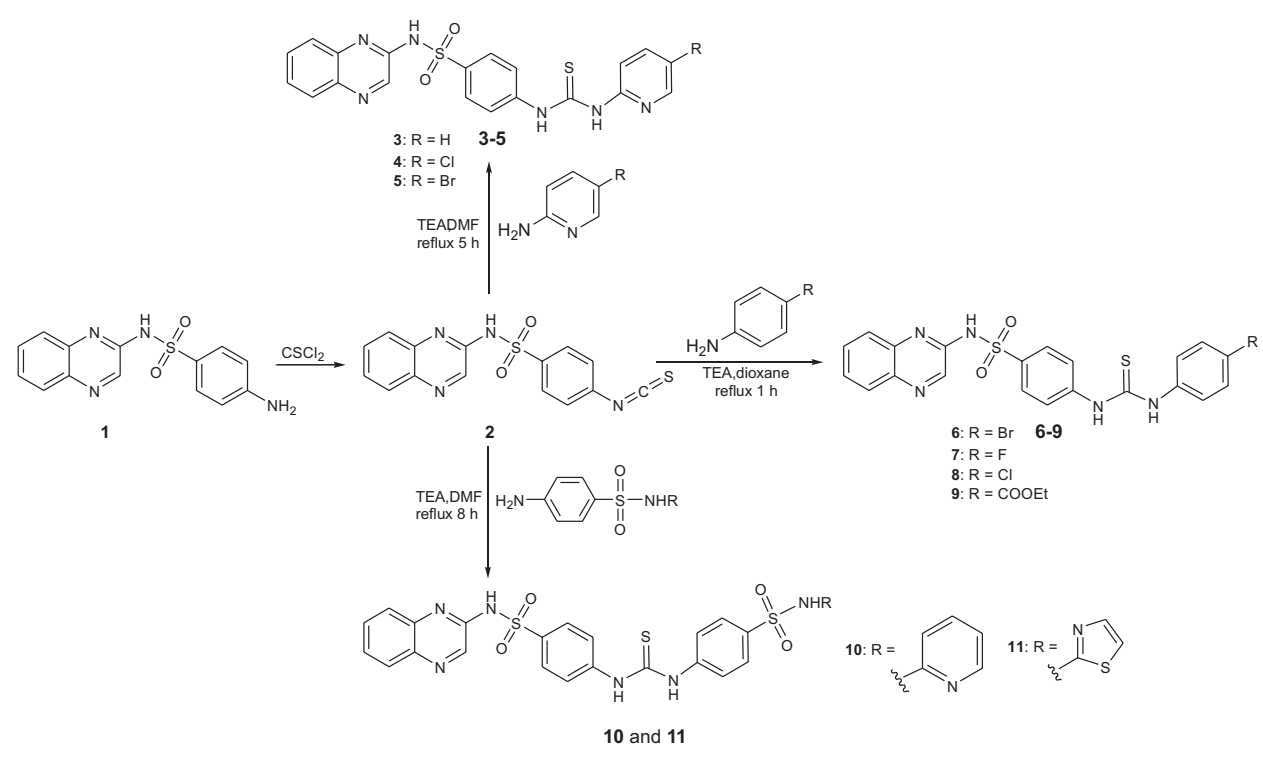

Scheme 1

Another sulfonamide molecule was incorporated into isothiocyanate $\mathbf{2}$ through its reaction with sulphapyridine and sulphathiazole and compounds $\mathbf{1 0}$ and $\mathbf{1 1}$ were obtained, respectively (Scheme 1).

\section{In vitro anticancer activity}

Newly synthesized compounds were evaluated for their in vitro cytotoxic activity against a human liver cancer cell line (HEPG2). Doxorubicin, one of the most effective anticancer agents, was used as the reference drug. The relationship between the surviving fraction and drug concentration was plotted to obtain the survival curve of the liver cancer cell line. The response parameter calculated was the $I C_{50}$ value, which corresponds to the concentration required for $50 \%$ inhibition of cell viability. Table III shows the in vitro anticancer activity of the synthesized compounds, which exhibited significant activity compared to the reference drug. It was found that in the negative control, the solvent had no effect on the cells as the surviving fraction was 1.00, all the tested compounds showed lower $I C_{50}$ than doxorubicin $\left(I C_{50}=71.8 \mu \mathrm{mol} \mathrm{L}-1\right)$, with compound $9\left(I C_{50}=15.6 \mu \mathrm{mol} \mathrm{L}^{-1}\right)$ being the most potent in this screening. This might be due to incorporation of ethylbenzoate group since the corresponding halogenated compounds 6 , 7 and 8 exhibited lower activity $\left(I C_{50}=31.2-39.6 \mu \mathrm{mol} \mathrm{L}^{-1}\right)$. Incorporation of pyridine moiety yielding moderate activity in compound $3\left(I_{50}=30.0 \mu \mathrm{mol} \mathrm{L}^{-1}\right)$ and this activity slightly increased to 27.6 and $28 \mu \mathrm{mol} \mathrm{L}^{-1}$, respectively, in compounds 4 and 5 containing halogenated pyridine moieties. Replacement of pyridine with sulfapyridine moiety in compound 10 yielded an increased activity $\left(I C_{50}=26.8 \mu \mathrm{mol} \mathrm{L}^{-1}\right)$ which was also in- 
M. M. Ghorab et al.: Synthesis, in vitro anticancer screening and radiosensitizing evaluation of some new 4-[3-(substituted)thioureido] -N-(quinoxalin-2-yl)benzenesulfonamide derivatives, Acta Pharm. 61 (2011) 415-425.

Table III. In vitro anticancer screening of synthesized compounds against the human liver cell line (HEPG 2)

\begin{tabular}{|c|c|c|c|c|c|c|}
\hline \multirow{3}{*}{ Compd. } & \multirow{3}{*}{ Control } & \multicolumn{4}{|c|}{ Compound concentration $(\mu \mathrm{mol} \mathrm{L}-1)$} & \multirow{3}{*}{$\begin{array}{c}I C_{50} \\
\left.(\mu \mathrm{mol} \mathrm{L})^{-1}\right)\end{array}$} \\
\hline & & 5 & 12.5 & 25 & 40 & \\
\hline & & \multicolumn{4}{|c|}{ Surviving fraction ${ }^{a}$} & \\
\hline Dox & 1.00 & $0.721 \pm 0.020$ & $0.546 \pm 0.020$ & $0.461 \pm 0.010$ & $0.494 \pm 0.030$ & 71.8 \\
\hline 2 & 1.00 & $0.934 \pm 0.002$ & $0.896 \pm 0.009$ & $0.848 \pm 0.028$ & $0.422 \pm 0.047$ & 45.0 \\
\hline 3 & 1.00 & $0.822 \pm 0.058$ & $0.646 \pm 0.026$ & $0.530 \pm 0.030$ & $0.447 \pm 0.022$ & 30.0 \\
\hline 4 & 1.00 & $0.876 \pm 0.032$ & $0.692 \pm 0.024$ & $0.456 \pm 0.023$ & $0.377 \pm 0.015$ & 27.6 \\
\hline 5 & 1.00 & $0.811 \pm 0.085$ & $0.657 \pm 0.016$ & $0.503 \pm 0.022$ & $0.375 \pm 0.043$ & 28.0 \\
\hline 6 & 1.00 & $0.921 \pm 0.008$ & $0.694 \pm 0.033$ & $0.621 \pm 0.032$ & $0.478 \pm 0.081$ & 35.2 \\
\hline 7 & 1.00 & $0.861 \pm 0.017$ & $0.745 \pm 0.015$ & $0.557 \pm 0.003$ & $0.407 \pm 0.027$ & 31.2 \\
\hline 8 & 1.00 & $0.818 \pm 0.044$ & $0.723 \pm 0.012$ & $0.622 \pm 0.014$ & $0.553 \pm 0.065$ & 39.6 \\
\hline 9 & 1.00 & $0.371 \pm 0.074$ & $0.298 \pm 0.033$ & $0.416 \pm 0.004$ & $0.394 \pm 0.021$ & 15.6 \\
\hline 10 & 1.00 & $0.748 \pm 0.025$ & $0.540 \pm 0.003$ & $0.433 \pm 0.076$ & $0.437 \pm 0.003$ & 26.8 \\
\hline 11 & 1.00 & $0.588 \pm 0.021$ & $0.507 \pm 0.058$ & $0.451 \pm 0.004$ & $0.406 \pm 0.016$ & 24.4 \\
\hline
\end{tabular}

a Mean \pm SEM, $n=3$.

Dox - doxorubicin

creased upon adding the sulfathiazole moiety in compound $\mathbf{1 1}\left(I C_{50}=24.4 \mu \mathrm{mol} \mathrm{L}^{-1}\right)$. Finally, it was observed that the isothiocyanate derivative 2 was the least potent in this study $\left(I_{50}=45 \mu \mathrm{mol} \mathrm{L}-1\right)$.

\section{Radiosensitizing evaluation}

The rationale for combining chemotherapy and radiotherapy is mainly based on two ideas, one being spatial cooperation, which is effective if chemotherapy is sufficiently active to eradicate subclinical metastases and if the primary local tumor is effectively treated by radiotherapy. In this regard, no interaction between radiotherapy and chemotherapy is required. The other idea is the enhancement of radiation effects. Cytotoxic agents can enhance radiation effects by direct enhancement of the initial radiation damage by incorporating drugs into DNA, inhibiting cellular repair, accumulating cells in a radiosensitive phase or eliminating radioresistant phase cells, eliminating hypoxic cells or inhibiting the accelerated repopulation of tumor cells. Consequently, the ability of the most active compounds $(9,10$ and 11) to enhance the cell killing effect of $\gamma$-irradiation was studied. From the results given in Tables III and IV, it was concluded that in the negative control, radiation alone had a low effect on the cells, compound 9 showed in vitro cytotoxic activity with the $I C_{50}$ value of $15.6 \mu \mathrm{mol} \mathrm{L}^{-1}$ when the cells were subjected to different concentrations of the compound alone. However, when the cells were subjected to the same concentrations of compound 9 and irradiated with a single dose of $\gamma$-radiation of $8 \mathrm{kGy}$, 
M. M. Ghorab et al.: Synthesis, in vitro anticancer screening and radiosensitizing evaluation of some new 4-[3-(substituted)thioureido] -N-(quinoxalin-2-yl)benzenesulfonamide derivatives, Acta Pharm. 61 (2011) 415-425.

Table IV. In vitro anticancer screening of compounds 9, 10 and 11 against the human liver cell line (HEPG2) in combination with $\gamma$-irradiation

\begin{tabular}{|c|c|c|c|c|c|c|c|}
\hline \multirow{2}{*}{\multicolumn{2}{|c|}{ Compd. Control }} & \multicolumn{5}{|c|}{$\begin{array}{l}\text { Compound concentration }\left(\mu \mathrm{mol} \mathrm{L}^{-1}\right)+\gamma \text {-irradiation }(8 \mathrm{kGy}) \\
\text { Surviving fraction }\end{array}$} & \multirow{2}{*}{$\begin{array}{c}I C_{50} \\
(\mu \mathrm{mol} \\
\left.\mathrm{L}^{-1}\right)\end{array}$} \\
\hline & & $0^{\mathrm{a}}$ & 5 & 12.5 & 25 & 40 & \\
\hline 9 & & $0.927 \pm 0$ & $0.27 \pm 0.08^{c}$ & $0.20 \pm 0.01^{c}$ & $0.31 \pm$ & $29 \pm$ & 4. \\
\hline 10 & 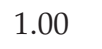 & $0.927 \pm$ & $0.55 \pm 0.01^{\mathrm{c}}$ & $0.34 \pm 0.02^{c}$ & $0.23 \pm 0$ & $0.13 \pm$ & 12 \\
\hline 11 & 1.00 & $0.927 \pm 0.02^{c}$ & $0.39 \pm 0.02^{c}$ & $0.31 \pm 0.01^{\mathrm{c}}$ & $0.25 \pm 0.01^{\mathrm{c}}$ & $0.21 \pm 0.01^{c}$ & 14.7 \\
\hline
\end{tabular}

a Irradiation (8 Gy) only.

b Mean \pm SEM, $n=3$.

c Significant difference from control group: $p<0.001$.

as shown in Table IV, the $I C_{50}$ value was synergistically decreased to $4.8 \mu \mathrm{mol} \mathrm{L}^{-1}$. Similarly, compounds 10 and $\mathbf{1 1}$ showed $I C_{50}$ values of 26.8 and $24.4 \mu \mathrm{mol} \mathrm{L}^{-1}$, respectively, when used alone, but their $I C_{50}$ values decreased to 12.3 and $14.7 \mu \mathrm{mol} \mathrm{L}^{-1}$, respectively, when the cells were treated with compounds $\mathbf{1 0}$ or $\mathbf{1 1}$ in combination with $\gamma$-irradiation. These results point to the conclusion that using a combination of compounds $\mathbf{9 ,} \mathbf{1 0}$ or $\mathbf{1 1}$ and ionizing radiation synergistically enhances growth inhibition of liver cancer cells compared to the use of each agent alone.

\section{CONCLUSIONS}

The above results allow the conclusion that administration of the tested compounds to the human liver (HEPG2) cell lines showed promising anticancer activity. The most potent compounds being4-(3-(4-ethylbenzoate)thioureido)- $N$-(quinoxalin-2-yl)benzenesulfonamide (9) $\left(I C_{50}=15.6 \mu \mathrm{mol} \mathrm{L}^{-1}\right), \mathrm{N}$-(pyridin-2-yl)-4-(3-(4-( $N$-quinoxalin-2-yl-sulfamoyl)phenyl)thioureido)benzenesulfonamide (10) $\left(I C_{50}=26.8 \mu \mathrm{mol} \mathrm{L}^{-1}\right)$ and $N$-(quinoxalin-2-yl)-4-(3-(4-( $N$-thiazol-2-ylsulfamoyl)phenyl)thioureido)benzenesulfonamide (11) $\left(I C_{50}=24.4 \mu \mathrm{mol} \mathrm{L}-1\right)$. Combining these compounds, at the same concentrations, with radiation enhances their activity. This indicates the importance of a combination therapy for cancer patients to reduce the side effects of both drugs and radiation.

\section{REFERENCES}

1. V. K. Tandon, D. B. Yadav, H. K. Maurya, A. K. Chaturvedi and P. K. Shukla, Design, synthesis, and biological evaluation of 1,2,3-trisubstituted-1,4-dihydrobenzo[ $g$ ]quinoxaline-5,10-diones and related compounds as antifungal and antibacterial agents, Bioorg. Med. Chem. 14 (2006) 6120-6126; DOI: 10.1016/j.bmc.2006.04.029.

2. S. Sehlstedt, P. Aich, J. Bergman, H. Vallberg, B. Nordén and A. Gräslund, Interactions of the antiviral quinoxaline derivative 9-OH-B220 \{2,3-dimethyl-6-(dimethylaminoethyl)-9-hydroxy-6H- 
-indolo-[2,3-b]quinoxaline\}with duplex and triplex forms of synthetic DNA and RNA, J. Mol. Biol. 278 (1998) 31-56; DOI: 10.1006/jmbi.1998.1670.

3. A. Carta, M. Loriga, G. Paglietti, A. Mattana, P. L. Fiori, P. Mollicotti, L. Mollicotti and S. Zanetti, Synthesis, anti-mycobacterial, anti-trichomonas and anti-candida in vitro activities of 2-substituted-6,7-difluoro-3-methylquinoxaline 1,4-dioxides, Eur. J. Med. Chem. 39 (2004) 195-203; DOI: 10.1016/j.ejmech.2003.11.008.

4. M. H. Fisher, A. Lusi and J. R. Egerton, Anthelmintic dihydroquinoxalino[2,3-b]quinoxalines, Eur. J. Med. Chem. 66 (1997) 1349-1352; DOI: 10.1002/jps.2600660942.

5. A. Budakoti, A. R. Bhat and A. Azam, Synthesis of new 2-(5-substituted-3-phenyl-2-pyrazolinyl)-1,3-thiazolino[5,4- $b$ ]quinoxaline derivatives and evaluation of their antiamoebic activity, Eur. J. Med. Chem. 44 (2009) 1317-1325; DOI: 10.1016/j.ejmech.2008.02.002.

6. R. S. Giri, H. M. Thaker, T. Giordano, J. Williams, D. Rogers, V. Sudersanam and K. K. Vasu, Design, synthesis and characterization of novel 2-(2,4-disubstituted-thiazole-5-yl)-3-aryl-3H-quinazoline-4-one derivatives as inhibitors of NF- $\mathrm{\kappa B}$ and AP-1 mediated transcription activation and as potential anti-inflammatory agents, Eur. J. Med. Chem. 44 (2009) 2184-2189; DOI: 10.1016/j. ejmech.2008.10.031.

7. J. S. Fisherman, B. L. Osborn and H. G. Chun, Chloroquinoxaline sulfonamide: A sulfanilamide antitumor agent entering clinical trials, Invest. New Drugs. 11 (1993) 1-9; DOI: 10.1007/BF00873904.

8. F. Mohamadi, M. M. Spees and G. B. Grindey, Sulfonylureas: A new class of cancer chemotherapeutic agents, J. Med. Chem. 35 (1992) 3012-3016; DOI: 10.1021/jm00094a013.

9. G. F. Rush, S. Rinzel, G. Boder, R. A. Heim, J. E. Toth and G. D. Ponsler, Effects of diarylsulfonylurea antitumor agents on the function of mitochondria isolated from rat liver and GC3/c1 cells, Biochem. Pharmacol. 44 (1992) 2387-2394; DOI: 10.1016/0006-2952(92)90684-B.

10. M. M. Ghorab, F. A. Ragab, H. I. Heiba, R. K. Arafa and E. M. El-Hossary, In vitro anticancer screening and radiosensitizing evaluation of some new quinolines and pyrimido[4,5-b]quinolines bearing a sulfonamide moiety, Eur. J. Med. Chem. 459 (2010) 3677-3684; DOI: 10.1016/j. ejmech.2010.05.014.

11. M. M. Ghorab, F. A. Ragab and M. M. Hamed, Design, synthesis and anticancer evaluation of novel tetrahydroquinoline derivatives containing sulfonamide moiety, Eur. J. Med. Chem. 44 (2009) 4211-4217; DOI: 10.1016/j.ejmech.2009.05.017.

12. M. M. Ghorab, F. A. Ragab, S. I. Alquasoumi, A. M. Alafeefy and S. A. Aboulmagd, Synthesis of some new pyrazolo[3,4-d]pyrimidine derivatives of expected anticancer and radioprotective activity, Eur. J. Med. Chem. 45 (2010) 171-178; DOI: 10.1016/j.ejmech.2009.09.039.

13. P. Skehan, R. Storeng, D. Scudiero, A. Monks, J. McMahon, D. Vistica, J. T. Warren, H. Bokesch, S. Kenny and M. R. Boyd, New colorimetric cytotoxicity assay for anticancer-drug screening, J. Natl. Cancer Inst. 82 (1990) 1107-1112; DOI: 10.1093/jnci/82.13.1107.

14. M. S. A. El-Gaby, A. M. Hussein, F. A. M. Abu-Shanab and M. A. M. Abdel Raheim, some hitherto unknown thiosemicarbazide, thiourea, bisthiourea, benzoazole derivatives bearing quinoxalin-2-yl moiety and evaluate their biological activity, Afinidad 60 (2003) 358-369; DOI: 2780.35400011978567.0050.

15. Y. Nishimura, Rationale for chemoradiotherapy, Int. J. Clin. Oncol. 9 (2004) 414-420; DOI: 10.1007/ s10147-004-0443-z. 


\section{Sinteza, in vitro antitumorsko ispitivanje i radiosenzitirajuće vrednovanje novih derivata 4-[3-(supstituiranih)tioureido]- $N$-(kinoksalin-2-il)benzensulfonamida}

MOSTAFA M. GHORAB, FATMA A. RAGAB, HELMY I. HEIBA, MARWA G. EL-GAZZAR i MOSTAFA G. EL-GAZZAR

Derivati sulfonamida i kinoksalina imaju raznoliko biološko djelovanje, između ostalog i antitumorsko djelovanje. U radu je opisana sinteza novih derivata tioureido sulfakinoksalina. Svim novim spojevima ispitano je antitumorsko djelovanje in vitro na humanoj staničnoj liniji jetre (HEPG 2). Svi ispitani spojevi pokazuju jači učinak nego referentni lijek doksorubicin. Najjači učinak imali su 4-(3-(4-etilbenzoat)tioureido)- $N$-(kinoksalin-2-il)benzensulfonamid (9) $\left(I_{50}=15,6 \mu \mathrm{mol} \mathrm{L}-1\right), N$-(piridin-2-il)-4-(3-(4-(N-kinoksalin-2-il-sulfamoil)fenil)tioureido)-benzensulfonamid (10) $\left(I C_{50}=26,8 \mu \mathrm{mol} \mathrm{L}^{-1}\right) \mathrm{i}$ $N$-(kinoksalin-2-il)-4-(3-(4-(N-tiazol-2-ilsulfamoil)fenil)tioureido)benzensulfonamid (11) $\left(I C_{50}=24,4 \mu \mathrm{mol} \mathrm{L}^{-1}\right)$, dok je $I C_{50}$ vrijednost bila 71,8 $\mu \mathrm{mol} \mathrm{L}^{-1}$. Najaktivniji spojevi $\mathbf{9}, \mathbf{1 0}$ i 11 evaluirani su kao radiosenzitirajuća sredstva nakon izlaganja spojeva $\gamma$-zračenju (8 kGy).

Ključne riječi: kinoksalin, sulfonamid, antitumorsko djelovanje, radiosenzitirajući učinak

Medicinal, Aromatic and Poisonous Plants Research Center (MAPPRC), College of Pharmacy King Saud University, Riyadh, Saudi Arabia

Department of Pharmaceutical Chemistry, Faculty of Pharmacy, Cairo University, Cairo, Egypt

Department of Drug Radiation Research, National Center for Radiation Research and Technology PO Box 29, Nasr City, Cairo, Egypt 